\title{
Eficiencia de dos intervenciones motivacionales para la deshabituación tabáquica en adolescentes (breve e intensiva) realizadas en Institutos de Educación Secundaria
}

\section{Efficiency of two motivational interventions for adolescent smokers (brief and intensive) conducted in High Schools}

\author{
Alejandro Pérez-Milena*; Ana Belén Navarrete- \\ Guillén ${ }^{\star *}$; María InMACUlada Mesa-Gallardoo**; Rocío \\ Martínez Pérez ${ }^{\star \star \star *}$; Francisco JaVier LeaL-HeLmLING ${ }^{\star \star \star \star *}$; \\ Carlos Pérez-Fuentes*
}

\author{
* Centro de Salud El Valle (Jaén), Servicio Andaluz de Salud. Grupo de \\ Estudio del Adolescente, Sociedad Andaluza de Medicina Familiar y \\ Comunitaria \\ ** Centro de Salud de Torredonjimeno (Jaén), Servicio Andaluz de Salud.

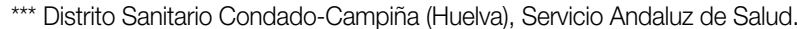 \\ Grupo de Estudio del Adolescente, Sociedad Andaluza de Medicina Familiar \\ y Comunitaria \\ ${ }^{\star \star \star \star}$ Centro de Salud Bollullos del Condado (Huelva), Servicio Andaluz de \\ Salud. Grupo de Estudio del Adolescente, Sociedad Andaluza de Medicina \\ Familiar y Comunitaria

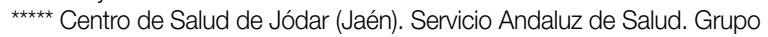 \\ de Estudio del Adolescente, Sociedad Andaluza de Medicina Familiar y \\ Comunitaria
}

\author{
Enviar correspondencia a: \\ Alejandro Pérez Milena. \\ Centro de Salud EI Valle. \\ C/ Ronda Alcalde García Segovia, 9. \\ 23009 Jaén. \\ Tel. 953313177 \\ alejandro.perez.milena.sspa@juntadeandalucia.es
}

\section{Resumen}

Se desea determinar la eficiencia de dos intervenciones motivacionales en adolescentes, basadas en el uso de disonancias cognitivas, para conseguir el cese del tabaquismo. Se realiza un estudio experimental aleatorizado multicéntrico en 5 Institutos de Educación Secundaria. Se proponen intervenciones individuales en los institutos participando adolescentes fumadores ( $\leq 20$ años) que desean dejar de fumar, con exclusión de embarazadas, trastornos graves de salud mental o uso de drogas antitabaco. Se recogió el consentimiento informado y un cuestionario previo (variables demográficas, consumo de tabaco/alcohol/drogas, apoyo familiar/social). Se desarrollan dos intervenciones por médicos de familia en el propio instituto mediante un muestreo aleatorio estratificado: intensiva (cuatro sesiones de 15 minutos, reducción progresiva del consumo) y breve (sesión única de 15 minutos, cese inmediato del consumo). Se confirma la abstinencia mediante cooximetría el primer, sexto y duodécimo mes tras la intervención, con análisis por intención de tratar. Participan 92 adolescentes, con edad media 15,4 $\pm 1,0$ años, sin diferencias al principio de las intervenciones: $82 \%$ fuman a diario, con baja dependencia (62\%) y motivación alta/moderada para el cese (88\%), 78\% consumen alcohol y $21 \%$ drogas. La función familiar y el apoyo social son normales en la mayoría. $47 \%$ recibe la intervención intensiva. Se consigue la abstinencia del 64\% $\pm 5,0$ el primer mes (20\% mejor en intervención intensiva, $\left.\mathrm{p}<0,05 \chi^{2}\right) ; 42 \% \pm 5,2$ el sexto mes y $27 \% \pm 4,6$ al año (sin diferencias). La intervención breve parece ser más eficiente, mientras que serian precisas más investigaciones para determinar el perfil del adolescente que se beneficiaría de una intervención intensiva.

Palabras clave: adolescente, tabaquismo, disonancia cognitiva, cese del tabaquismo. recibido: Noviembre 2011 aceptado: Mayo 2012

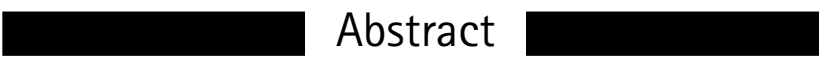

We set out to determine the efficiency of two motivational interventions (brief and intensive) in adolescent smokers, based on obtaining cognitive dissonance and seeking to help them stop smoking. A multicenter randomized experimental study was carried out at five high schools. Individual anti-smoking interventions were applied at the schools, the participants being adolescent smokers ( $\leq 20$ years) who wished to quit smoking. Exclusion criteria were use of anti-smoking drugs, severe psychiatric illness and pregnancy. Informed consent was obtained and a questionnaire recorded demographic variables and alcohol/tobacco/other drug use. Two motivational interventions were carried out at each school by GP, in accordance with a stratified randomization procedure: intensive (four sessions, progressive reduction of smoking) and brief (single session, immediate cessation of smoking). Smoking abstinence was confirmed by co-oximetry at 1, 6 and 12 months after the intervention, with analysis by intention to treat. A total of 92 adolescents participated, with a mean age of $15.4 \pm 1.0$ years; no differences at the beginning of the interventions: daily smokers accounted for $82 \%$ of the sample, with low dependence (62\%) and moderate-high motivation to quit smoking (88\%). Seventy-eight per cent used alcohol and $21 \%$ other drugs. Family functioning and social support were normal in the majority. $47 \%$ received the intensive intervention. Abstinence was achieved by $64 \% \pm 5.0$ by the first month $(20 \%$ better in intensive intervention), $42 \% \pm 5.2$ by the sixth month and $27 \% \pm 4.6$ by the twelfth month (without differences). The brief intervention appears to be more efficient, while more research is needed to determine the profile of those adolescents who would benefit from intensive intervention.

Key words: adolescent, smoking, cognitive dissonance, smoking cessation. 
$\mathrm{L}$ a Organización Mundial de la Salud considera el tabaquismo como uno de los principales problemas de la salud pública mundial, sobre todo en la población infantil y adolescente (Lando, Hipple, Muramoto, Klein, Prokhorov et al., 2010). En España los adolescentes forman un subgrupo poblacional de especial riesgo por la alta prevalencia del consumo y sus caracteristicas (ESTUDES, 2010; Infante y Rubio-Colavida, 2004; Pérez-Milena et al., 2007). Los resultados de la Encuesta Estatal sobre el uso de drogas en Enseñanzas Secundarias del Plan Nacional de Drogas (ESTUDES, 2010) muestran una reducción del consumo de tabaco en adolescentes de 14 a 18 años respecto a años previos; pese a ello, casi un 45\% de los adolescentes españoles han fumado alguna vez y el $15 \%$ lo hacen de forma diaria, con un inicio precoz del consumo y un mayor consumo entre las mujeres. Esta encuesta muestra, además, que las vías por las que los jóvenes preferirian recibir información sobre drogas son, preferentemente, los profesionales sanitarios e internet.

La intervención para conseguir el cese del consumo del tabaquismo en la población general está recogida en multitud de guías publicadas por instituciones oficiales y sociedades científicas (SEMFYC, 2007; Moreno, 2008; NICE, 2008; Ramos y Mayayo, 2007), incluyendo diversas actividades ya establecidas en la práctica diaria: el consejo sanitario, la intervención breve, la intervención intensiva y/o el tratamiento farmacológico (Moreno, 2008; NICE, 2008). Sin embargo, no existe información contrastada sobre la efectividad de estas terapias en la población adolescente (Barrueco, Gómez, Torrecilla, Pérez y Bartolomé, 2007; Hettema y Hendricks, 2010). Los programas escolares destinados a niños y jóvenes, muchos de ellos dirigidos a la prevención, son las intervenciones más ampliamente utilizadas y cuentan con una amplia experiencia; sin embargo, en los últimos años se ha cuestionado su eficacia (Barrueco et al., 2007). Por otro lado, las intervenciones individuales realizadas en adultos no tienen igual validez sobre los adolescentes fumadores dadas las diferencias en los patrones de consumo de tabaco, el estilo de vida y las actitudes hacia los servicios sanitario de este grupo etario (Grimshaw y Stanton, 2007; Johnson et al, 2009). Existe una gran variabilidad en la atención prestada a estos adolescentes fumadores así como una falta de información contrastada acerca de la efectividad de los protocolos de intervención para dejar de fumar (Barrueco et al., 2007; Grimshaw y Stanton, 2007).

Frente a las pocas evidencias existentes en este campo, la realidad es que hay un importante porcentaje de adolescentes fumadores que quieren dejar de consumir tabaco (PérezMilena et al., 2006). Este porcentaje es más elevado que en la población adulta, lo que puede ser empleado como un elemento positivo que ayude a conseguir la abstinencia en estas edades. La entrevista motivacional ha sido empleada en este tipo de intervenciones pero sin alcanzar evidencias sobre su utilidad, dada la dificultad para evaluar y comparar los resultados de los estudios sobre las diferentes intervenciones motivacionales entre sí, debido a la variabilidad y heterogeneidad de los mismos (Douglas et al., 2010; Grimshaw y Stanton, 2007; Hettema y Hendricks, 2010; Pérez-Trullén, Herrero, Clemente y Escosa, 2004; Piñeiro, Míguez y Beco- ña, 2010). Los estudios sobre intervenciones motivacionales para conseguir la deshabituación tabáquica en adolescentes sugieren que se trata de una técnica útil para esta población (Heckman, Egleston y Hofman, 2010; Hettema y Hendricks, 2010) aunque no hay suficientes evidencias (Rivera, Villouta e llabaca, 2008). Dentro de las herramientas que oferta la entrevista motivacional, el empleo de disonancias cognitivas para el cese del tabaquismo puede tener una importante eficacia (Heckman et al., 2010; Hettema y Hendricks, 2010; Kleinjan, Van den Eijnden y Engels, 2009).

Las características del tabaquismo en adolescentes, su grave potencial de daño en el fumador joven y su necesario abordaje son las causas del presente trabajo, donde se plantea como objetivo conocer la efectividad de dos tipos de intervenciones motivacionales, una breve y otra intensiva, basadas en el uso de las disonancias cognitivas para el cese del hábito tabáquico en adolescentes fumadores, sin emplear ningún recurso farmacológico.

\section{Método}

\section{Participantes}

La población diana son los adolescentes fumadores escolarizados en los institutos de Educación Secundaria de Andalucía. Se elige como población de estudio el alumnado perteneciente a dos institutos urbanos (IES Jabalcuz y El Valle, en Jaén capital) y a tres rurales (Torredonjimeno y Jódar en Jaén, y Bollullos del Condado en Huelva), durante los cursos escolares 2009/10 y 2010/11. Se incluyen adolescentes con una edad $\leq 20$ años y fumadores habituales (definido como un consumo de al menos un cigarrillo semanal durante los últimos seis meses) (Grimshaw y Stanton, 2007). Los criterios de exclusión son: deficiencia mental, patología psiquiátrica grave, embarazo, uso concomitante de tratamiento farmacológico para la deshabituación tabáquica y/o negativa del adolescente 0 de sus padres/tutores a participar en el estudio. La captación de los adolescentes fue realizada por los orientadores/as de cada instituto. Se realizó un muestreo aleatorio simple, estratificado por cada centro educativo, con asignación ciega a cada grupo (mediante el programa Epidat 3.1), calculando un tamaño de muestra de 42 sujetos por grupo mediante una prueba $\chi^{2}$ unilateral para dos muestras independientes, con una potencia del 80\%, un nivel de significación del $5 \%$ y asumiendo que la diferencia de efectividad de ambas intervenciones es de un 15\%.

\section{Intervenciones}

El diseño del estudio es experimental, multicéntrico y aleatorizado, comparando la abstinencia tabáquica continua obtenida mediante dos intervenciones basadas en la obtención de disonancias cognitivas de los adolescentes fumadores a partir de un registro escrito aportado por el propio fumador.

En un primer grupo (intervención intensiva) se realizaron cuatro sesiones semanales de 15 minutos de duración cada 
una, con una reducción progresiva de un 30\% del consumo en las dos primeras visitas (en base a un autorregistro diario del consumo), fijando una fecha para dejar de fumar en la $3^{\text {a }}$ visita y con una $4^{\text {a }}$ visita de refuerzo. En el segundo grupo (intervención breve) se realiza una sola sesión de 15 minutos donde se dan consejos prácticos para el cese del hábito tabáquico y se fija una fecha para el cese del consumo. El guión de cada intervención se muestra en la tabla 1. Se envían recordatorios a todos los adolescentes mediante SMS el día que dejan de fumar, el día previo y la semana posterior, así como e-mail mensuales durante un año. Las intervenciones individuales se realizaron en los institutos, durante la jornada escolar, por parte de seis médicos de familias adiestrados previamente en este tipo de intervenciones (Miller y Rollnick, 1999). Se realizaron antes de las intervenciones unas reuniones formativas, dirigidas por un médico de familia experto en entrevista clínica, para homogeneizar las actuaciones.

Tabla 1. Guiones para la realización de las intervenciones motivacionales individuales intensiva y breve para la deshabituación tabáquica

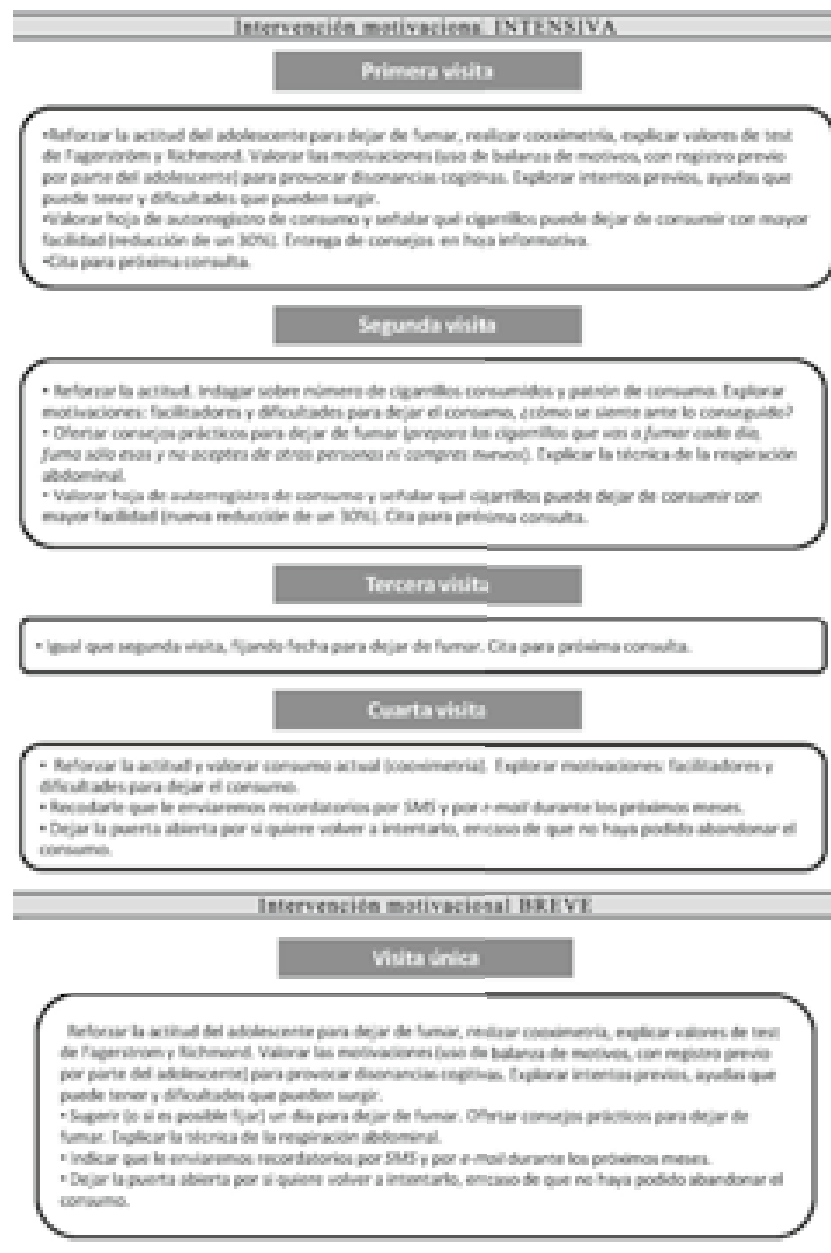

\section{Medidas e instrumentos}

El resultado principal es la abstinencia continua, definida como el cese del consumo de tabaco a los doce meses de la fecha fijada por el adolescente para dejar de fumar (Grimshaw y Stanton, 2007; Hughes et al., 2003). Se valora mediante abstinencia autodeclarada y validada con cooximetría (medición de monóxido de carbono en aire espirado) mediante un cooxímetro modelo Smoke Check, que clasifica los resultados en cuatro valores: bajo (0-6ppm), moderado (7-12ppm), alto (12-20ppm) y muy alto (>20ppm). El punto de corte para diferenciar fumadores de no fumadores está establecido para este modelo en 6 ppm de monóxido de carbono. También se valora la prevalencia puntual de la abstinencia a los 30 días tras el momento de cese del consumo y la abstinencia continua a los seis meses. Al inicio del estudio se recoge edad, sexo y patrón de consumo de tabaco, alcohol y drogas clasificado como intermitente, en fin de semana o diario (Pérez-Milena et al., 2007), así como el consumo de alcohol como unidades de bebida estándar por semana y de tabaco como número de cigarrillos al día. Se administran el test de Richmond para valorar la motivación (con una motivación nula o baja entre cero y 3 puntos, dudosa entre 4 y 5, moderada de 6 a 7, y alta a partir de 8) y el test de Fagerström para valorar la dependencia a la nicotina (dependencia baja en valores $<4$, moderada entre 4 y 7, y alta si obtiene más de 7 puntos). La función familiar se valora mediante la aplicación del test de Apgar familiar, validado para la población española (Bellón, Delgado, Luna y Lardelli, 1996a), que mide la impresión subjetiva del adolescente sobre la funcionalidad de su familia así como su integración en ella, considerando la existencia de disfunción grave con valores entre 0 a 3, disfunción leve de 4 a 7, y función normal a partir de 8 . El apoyo social se valora con el test DUKE-UNC, un cuestionario autoadministrado validado y fiable para la población general (Bellón, Delgado, Luna y LardeIli, 1996b), diferenciado el apoyo afectivo y el confidencial; se considera que el sujeto encuestado tiene un apoyo social bajo cuando el resultado está por debajo de 33 (15 para el apoyo afectivo y 18 para el confidencial).

\section{Aspectos éticos}

El estudio ha contado con la aprobación previa del Comité de Ética de la Investigación Científica de la provincia de Jaén, así como del Consejo Escolar y Claustro de Profesores de cada instituto. Se entregó información por escrito sobre la investigación y se solicitó la firma de un consentimiento informado por parte del adolescente para proporcionar voluntariedad, información y compromiso. Tanto el diseño como el desarrollo del trabajo se ajustan a las normas de buena práctica clínica (Ley 41/2002 de autonomía del paciente) y a la protección de datos personales (Ley 15/1999 de protección de datos y ley 14/2007 de Investigación Biomédica).

\section{Análisis estadístico}

Se ha realizado un análisis de los datos obtenidos mediante intención de tratar, usando el programa SPSS v15. Se aplicó el test de Kolmogorov-Smirnov para comprobar la normalidad de los datos, que se muestran como media y su error estándar de la media o de la proporción. Se valora la fiabilidad de los cuestionarios empleados (Fagerström, Richmond, Apgar familar y DUKE-UNC) mediante el índice de consistencia interna alfa de Cronbach, calculado con el 
método de la varianza de los ítems. Se propone un nivel de significación estadística con $p<0,05$, aplicándose el test de la $t$ de Student para comparación de medias y el test $\chi^{2}$ para la comparación de proporciones para valorar la similitud entre ambos grupos. Para el cese del consumo de tabaco se relacionan la respuesta verbal del adolescente con los valores de la cooximetría mediante una tabla de contingencia (test $\chi^{2}$ ) y la concordancia con el índice kappa; también se calculan la sensibilidad, la especificidad, los valores predictivos y la razón de verosimilitud positiva. Las diferencias en la abstinencia entre cada grupo se valoraron mediante una análisis bivariado con el test $\chi^{2}$ y se calcularon los valores de la odds ratio (OR) y el número necesario de pacientes a tratar (NNT) para cada intervención. Por último, se realizó un multivariante (mediante regresión logística) incluyendo todas las variables recogidas para conocer cuáles de ellas se relacionan con la abstinencia continua al año de la intervención. El modelo final se ha construido siguiendo un algoritmo hacia atrás, estableciendo como criterio estadístico para la eliminación de variables la cercanía al valor cero de los coeficientes encontrados y la no significación de cada variable. Para evaluar la bondad del ajuste del modelo final se ha utilizado el test de Hosmer-Lemeshow.

\section{Resultados}

Han participado un total de 92 adolescentes, siendo excluido sólo uno por estar usando parches de nicotina y sin cambios de grupo durante el seguimiento (figura 1). La intervención intensiva se realizó en 43 adolescentes y la intervención breve en los restantes 48 . Hay una pérdida en el grupo de intervención intensiva y dos en el de intervención breve.

\section{Características sociodemográficas}

Las principales variables medidas al inicio del estudio no presentaron diferencias relevantes entre grupos (tabla 2).

\section{Variables personales}

La media de edad es de 15,4 años $[ \pm 1,0]$ con un $51 \%$ de hombres repartidos de forma desigual entre los dos grupos (tabla 2, p<0,10 test $\chi^{2}$ ). Un 18\% tienen alguna enfermedad crónica y un $12 \%$ toman medicación continua (sobre todo patología respiratoria), siendo más frecuente en el grupo de intervención breve (tabla 2, $\mathrm{p}<0,05$ test $\chi^{2}$ ).

\section{Variables de consumo de tabaco}

La media de edad de inicio del consumo de tabaco se sitúa en 12,9 años $[ \pm 0,2]$, con un consumo mayoritariamente diario $(82 \%)$ de unos 8,3 cigarrillos $[ \pm 0,6]$ al día, incrementándose durante el fin de semana $(15,9$ cigarrillos $[ \pm 1,0]$; $\mathrm{p}<0,05$ test $t$ Student). El test de Fagerström muestra una puntuación media de $3,1[ \pm 0,2]$, con un $62 \%$ de adolescentes que puntúan en la categoria de baja dependencia a la nico-
Tabla 2. Valores de las variables personales, de consumo y sociofamiliares de los adolescentes en cada grupo antes de las intervenciones.

\begin{tabular}{|c|c|c|}
\hline & $\begin{array}{l}\text { Intervención } \\
\text { INTENSIVA }\end{array}$ & $\begin{array}{l}\text { Intervención } \\
\text { BREVE }\end{array}$ \\
\hline Tamaño de muestra & $43(47 \%)$ & $48(53 \%)$ \\
\hline \multicolumn{3}{|l|}{ Variables personales } \\
\hline Zona urbana & $42 \% \pm 7,5$ & $44 \% \pm 7,2$ \\
\hline Sexo Mujer (1) & $40 \%$ & $58 \%$ \\
\hline Edad (años) & $15,2 \pm 0,16$ & $15,7 \pm 0,24$ \\
\hline Enfermedad crónica (2) & $9 \%$ & $25 \%$ \\
\hline Medicación crónica (3) & $5 \%$ & $19 \%$ \\
\hline \multicolumn{3}{|l|}{ Variables de consumo de tabaco } \\
\hline $\begin{array}{l}\text { Patrón tabaquismo } \\
\text { Intermitente/Fin de semana/Diario }\end{array}$ & $16 \% / 0 \% / 84 \%$ & $13 \% / 6 \% / 81 \%$ \\
\hline Edad de comienzo (años) & $12,56 \pm 0,27$ & $13,19 \pm 0,26$ \\
\hline $\begin{array}{r}\text { Consumo en cigarrillos/dia } \\
\text { Diario } \\
\text { Fin de semana } \\
\text { Media global }\end{array}$ & $\begin{array}{c}9,5 \pm 0,84 \\
17,91 \pm 1,55 \\
14,48 \pm 1,16 \\
\end{array}$ & $\begin{array}{c}7,13 \pm 0,69 \\
14,17 \pm 1,18 \\
11,16 \pm 0,93\end{array}$ \\
\hline Intento previo de abstinencia & $79 \%$ & $73 \%$ \\
\hline $\begin{array}{l}\text { Test de Fagerström } \\
\text { Puntuación media } \\
\text { Dependencia baja/moderada/alta (4) }\end{array}$ & $\begin{array}{c}3,47 \pm 0,32 \\
49 \% / 51 \% / 0 \%\end{array}$ & $\begin{array}{c}2,73 \pm 0,29 \\
73 \% / 25 \% / 2 \%\end{array}$ \\
\hline $\begin{array}{l}\text { Test de Richmond } \\
\text { Puntuación media } \\
\text { Motivación dudosa/moderada/alta }\end{array}$ & $\begin{array}{c}6,88 \pm 0,22 \\
14 \% / 56 \% / 30 \%\end{array}$ & $\begin{array}{c}6,69 \pm 0,24 \\
20 \% / 44 \% / 36 \%\end{array}$ \\
\hline \multicolumn{3}{|c|}{ Variables de consumo de alcohol y drogas } \\
\hline $\begin{array}{l}\text { Alcohol } \\
\text { Edad de comienzo (años) } \\
\text { Consumo en UBE/semana }\end{array}$ & $\begin{array}{c}79 \% \\
13,41 \pm 0,23 \\
6,74 \pm 1,05\end{array}$ & $\begin{array}{c}77 \% \\
13,67 \pm 0,25 \\
7,21 \pm 1,03\end{array}$ \\
\hline $\begin{array}{l}\text { Drogas ilegales (5) } \\
\text { Edad de comienzo (años) }\end{array}$ & $\begin{array}{c}33 \% \\
14,29 \pm 0,30\end{array}$ & $\begin{array}{c}10 \% \\
13,40 \pm 0,60\end{array}$ \\
\hline \multicolumn{3}{|l|}{ Función familiar y apoyo social } \\
\hline $\begin{array}{l}\text { Test Apgar familiar } \\
\text { Puntuación media } \\
\text { Disfunción familiar leve/grave }\end{array}$ & $\begin{array}{c}6,9 \pm 0,38 \\
26 \% / 12 \%\end{array}$ & $\begin{array}{l}7,80 \pm 0,30 \\
11 \% / 7 \%\end{array}$ \\
\hline $\begin{array}{r}\text { Test DUKE-UNC } \\
\text { Puntuación media } \\
\text { Apoyo social normal } \\
\text { Apoyo afectivo normal } \\
\text { Apoyo confidencial normal }\end{array}$ & $\begin{array}{c}45,42 \pm 0,89 \\
98 \% \\
95 \% \\
88 \%\end{array}$ & $\begin{array}{c}47,20 \pm 1,01 \\
98 \% \\
91 \% \\
98 \%\end{array}$ \\
\hline $\begin{array}{l}\text { Nota. } \\
\text { (1) chi } 0,121 \text { con } 1 \text { grado de libertad } \\
\text { (2) } \text { chi }^{2} 0,716 \text { con } 1 \mathrm{gl}, p=0,050 \text { test } \chi^{2} \\
\text { (3) } \text { chi }^{2} 2,095 \text { con } 1 \mathrm{gl}, p=0,039 \text { test } \chi^{2} \\
\text { (4) chi } \text { ch }^{2}, 205 \text { con } 1 \mathrm{gl}, p=0,027 \text { test } \chi^{2} \\
\text { (5) chi } 0,291 \text { con } 1 \mathrm{gl}, p=0,009 \text { test } \chi^{2}\end{array}$ & test $\chi^{2}$ & \\
\hline
\end{tabular}

tina, más frecuente en el grupo de intervención breve (tabla $2, p<0,05$ test $\chi^{2}$ ). El test de Richmond puntúa de media 6,8 $[ \pm 0,2]$ con una motivación moderada en un $50 \%$ y alta en un $33 \%$. Ambos tests presentan una fiabilidad en rango aceptable ( $\alpha$ de Cronbach $=0,867$ para el test de Fagerström y $\alpha$ de Cronbach =0,801 para el test de Richmond). El 76\% han realizado una media de $1,6[ \pm 0,2]$ intentos previos para dejar de fumar. Los valores iniciales de las cooximetrias se sitúan en valores bajos (68\%) y medios (24\%) de CO espirado, sin diferencias entre grupos.

\section{Variables de consumo de alcohol y drogas ilegales}

Un 78\% consumen alcohol (79\% en fin de semana), con una edad de inicio de 13,5 años $[ \pm 0,2]$. Las unidades de bebida estándar semanales de cada tipo de bebida alcohólica son: cerveza $1,3[ \pm 0,3]$, vino $0,3[ \pm 0,1]$, combinados $5,0[ \pm 0,6]$ y licores $0,4[ \pm 0,1]$. Un $21 \%$ ha probado alguna vez otro tipo 
de drogas (más frecuente en el grupo de intervención intensiva, 33\% frente a 10\%; $p<0,01$ test $\chi^{2}$ ), con una edad de inicio de 14,1 años $[ \pm 0,3]$, indicando únicamente el consumo de cannabis.

\section{Función familiar y apoyo social}

El test de Apgar familiar tiene una puntuación media de $7,4[ \pm 0,3]$ y muestra una percepción normal de la función familiar en un $72 \%$ de los adolescentes, con sólo un $9 \%$ de disfunciones graves. El test de DUKE-UNC muestra una media de 46,3 puntos $[ \pm 0,7]$, con un apoyo social normal en un $98 \%$ de los menores (93\% en apoyo afectivo y $90 \%$ en apoyo confidencial). Ambos cuestionarios presentan una buena fiabilidad tras calcular el índice de consistencia interna $(\alpha$ de Cronbach $=0,893$ para el test de Apgar familiar y $\alpha$ de Cronbach $=0,954$ para el test DUKE-UNC).

\section{Resultado de las intervenciones}

El seguimiento y resultado obtenido en cada grupo de intervención se exponen en la figura 1. No hay diferencias entre los médicos que realizan las intervenciones ni entre los diferentes institutos. Al mes de las intervenciones motivacionales, el 64\% $( \pm 5,0)$ de los adolescentes dejan de fumar, 74\% $( \pm 6,6)$ en el grupo de intervención intensiva y $54 \%( \pm 7,2)$ en el grupo de intervención breve $\left(\chi^{2}=4,025,1 \mathrm{gl}, \mathrm{p}=0,045\right)$. A los seis meses la abstinencia disminuye al $42 \%( \pm 5,2)$

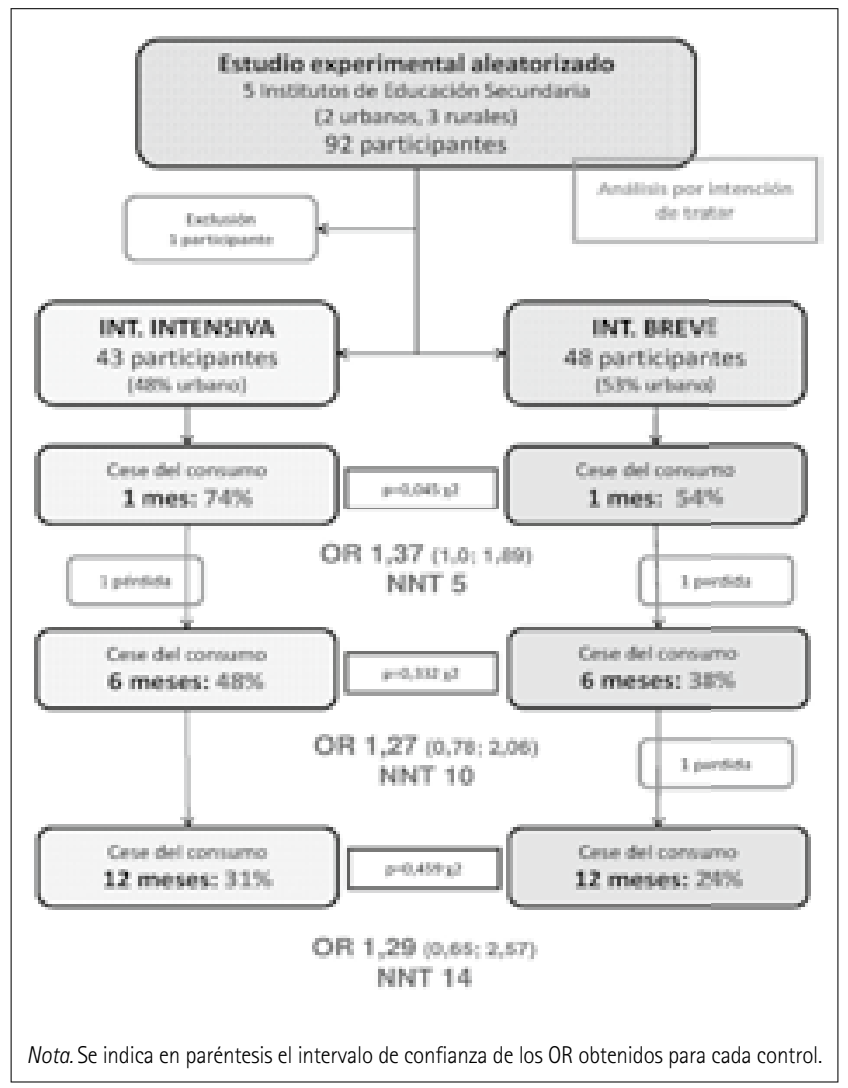

Figura 1. Diagrama de seguimiento de la efectividad de cada tipo de intervención motivacional para la deshabituación tabáquica según el momento de medición de la abstinencia tabáquica.
(48\% 7,6 en intervención intensiva y $38 \% \pm 7,0$ en intervención breve) $\left(\chi^{2}=0,940,1 \mathrm{gl}, \mathrm{p}=0,332\right)$ y a los doce meses el cese del consumo se mantiene en un $27 \%( \pm 4,6)(31 \% \pm 7,1$ intensiva y $24 \% \pm 6,3$ breve) $\left(\chi^{2}=0,548,1 \mathrm{gl}, p=0,459\right)$. Los valores de OR y NNT varían a lo largo del seguimiento (figura 1), apreciándose cómo disminuye la OR y aumenta el NNT conforme pasa el tiempo desde la fecha fijada para el cese del consumo.

Los valores de las cooximetrias se relacionan con la respuesta verbal de abstinencia, encontrando valores superiores a 6 ppm de $\mathrm{CO}$ sólo en adolescentes fumadores, mientras que el $100 \%$ de los adolescentes que reconocen mantener la abstinencia presentan valores iguales o inferiores a 6 ppm CO en aire espirado. Los valores de los índices kappa encontrados en cada momento de medición de la abstinencia son bajos, sin embargo la sensibilidad y los valores predictivos negativos son muy elevados (tabla 3 ).

Tabla 3. Relación entre la confirmación verbal de la abstinencia y la cooximetría realizada tras las intervenciones en cada momento de control del cese del tabaquismo.

\begin{tabular}{|c|c|c|c|c|}
\hline & & \multicolumn{2}{|c|}{ Abstinencia verbal } & \\
\hline & & Deja de fumar & Continua fumando & \\
\hline $\begin{array}{l}\text { Control al mes } \\
\text { (Cooximetria) }\end{array}$ & $\begin{array}{l}\leq 6 \mathrm{ppm} \mathrm{CO} \\
\geq 7 \mathrm{ppm} \mathrm{CO}\end{array}$ & $\begin{array}{l}98 \% \\
2 \%\end{array}$ & $\begin{array}{l}79 \% \\
21 \% \\
\end{array}$ & $\begin{array}{l}\text { chi }^{2} \text { 9,962 (2 gl) } \\
\mathrm{p}=0,002 \text { test } \chi^{2}\end{array}$ \\
\hline \multicolumn{5}{|c|}{$\begin{array}{c}\text { Índice kappa } 0,233( \pm 0,085)(\mathrm{p}=0,002) \\
\text { Sensibilidad } 0,98[0,95 ; 1,00] ; \text { Especificidad } 0,21[0,13 ; 0,29] \\
\text { Valor Predictivo Positivo } 0,55[0,48 ; 0,63] ; \text { Valor Predictivo Negativo } 0,91[0,80 ; 1,00] \\
\text { Razón de verosimilitud positiva } 1,24[1,12 ; 1.38]\end{array}$} \\
\hline & & \multicolumn{2}{|c|}{ Abstinencia verbal } & \\
\hline & & Deja de fumar & Continua fumando & \\
\hline $\begin{array}{l}\text { ontrol } 6^{\circ} \text { mes } \\
\text { ooximetria) }\end{array}$ & $\begin{array}{l}\leq 6 \mathrm{ppm} \mathrm{CO} \\
\geq 7 \mathrm{ppm} \mathrm{CO}\end{array}$ & $\begin{array}{l}100 \% \\
0 \%\end{array}$ & $\begin{array}{l}75 \% \\
25 \% \\
\end{array}$ & $\begin{array}{l}\text { chi }^{2} 11,104(2 \mathrm{gl}) \\
\mathrm{p}=0,001 \text { test } \chi^{2}\end{array}$ \\
\hline \multicolumn{5}{|c|}{$\begin{array}{c}\text { Índice kappa } 0,220( \pm 0,059)(\mathrm{p}=0,001) \\
\text { Sensibilidad } 0,99[0,97 ; 1,00] ; \text { Especificidad } 0,25[0,17 ; 0,33] \\
\text { Valor Predictivo Positivo } 0,57[0,50 ; 0,64] ; \text { Valor Predictivo Negativo } 0,96[0,89 ; 1,00] \\
\text { Razón de verosimilitud positiva } 0,76[0,68 ; 0,85]\end{array}$} \\
\hline & & \multicolumn{2}{|c|}{ Abstinencia verbal } & \\
\hline & & Deja de fumar & Continua fumando & \\
\hline $\begin{array}{l}\text { Control } 12^{\circ} \text { mes } \\
\text { (cooximetria) }\end{array}$ & $\begin{array}{l}\leq 6 \mathrm{ppm} \mathrm{CO} \\
\geq 7 \mathrm{ppm} \mathrm{CO}\end{array}$ & $\begin{array}{l}100 \% \\
0 \%\end{array}$ & $\begin{array}{l}70 \% \\
30 \% \\
\end{array}$ & $\begin{array}{l}\text { chi }^{2} 8,763(2 \mathrm{gl}) \\
\mathrm{p}=0,013 \text { test } \chi^{2}\end{array}$ \\
\hline \multicolumn{5}{|c|}{$\begin{array}{c}\text { Índice kappa } 0,218( \pm 0,043)(p=0,002) \\
\text { Sensibilidad } 0,70[0,61 ; 0,79] ; \text { Especificidad } 0,01[0,01 ; 0,03] \\
\text { Valor Predictivo Positivo } 0,41[0,34 ; 0,49] ; \text { Valor Predictivo Negativo } 0,03[0,01 ; 0,09] \\
\text { Razón de verosimilitud positiva } 0,71[0,62 ; 0,81]\end{array}$} \\
\hline
\end{tabular}

Nota. (gl) grados de libertad. Se indican entre corchetes los intervalos de confianza al 95\% de sensibilidad, especificidad, valores predictivos y razón de verosimilitud.

\section{Variables relacionadas con la abstinencia continua}

Se obtiene un modelo explicativo de la abstinencia continua (tabla 4) tras realizar una regresión logística multivariante considerando la abstinencia continua al año tras la intervención como variable dependiente. Se aprecia que la edad del adolescente se asocia al hecho de continuar fumando: por cada año de edad que tiene el adolescente cuando se realiza la intervención, hay un incremento del riesgo en 1,53 odds para continuar fumando transcurrido un año (el nivel de significación es superior a 0,05 pero el intervalo de confianza de la OR excluye el valor 1, por lo que clínicamente aporta diferencias relevantes). La otra variable que alcanza 
Tabla 4. Modelo explicativo de la abstinencia continua a los doce meses obtenida mediante regresión lineal múltiple.

\begin{tabular}{lccccc}
\hline \multirow{2}{*}{ Variable } & \multicolumn{5}{c}{ Abstinencia continua a los doce meses } \\
\cline { 2 - 6 } & Coeficiente & OR & $\begin{array}{c}\text { Intervalo de } \\
\text { confianza 95\% }\end{array}$ & Chi $^{2}$ & $\begin{array}{c}\text { Nivel de } \\
\text { significación }\end{array}$ \\
\hline Constante & $-6,78514$ & -- & -- & $-1,172$ & 0,2411 \\
\hline Edad & 0,654 & 0,192 & {$[0,099 ; 0,413]$} & 1,831 & 0,0671 \\
\hline Sexo (mujer) & 1,036 & 0,228 & {$[0,047 ; 2,108]$} & 1,091 & 0,2753 \\
\hline Test Richmond & $-0,350$ & 0,705 & {$[0.486 ; 0,985]$} & $-1,958$ & 0,0502 \\
\hline Test Fagerström & 0,058 & 0,106 & {$[0,066 ; 1,674]$} & 0,252 & 0,8008 \\
\hline
\end{tabular}

Nota. Test de Hosmer-Lemeshow con $\operatorname{chi}^{2} 1,177(p=0,555)$

la significación estadística en este modelo es la puntuación del test Richmond, medida como variable cuantitativa. Así, por cada punto más obtenido en este cuestionario, hay una disminución del riesgo de continuar fumando al año de la intervención en 1,41 odds. No se aprecia en ningún modelo una relación entre el médico que realiza la intervención, el instituto, la zona urbana/rural o el tipo de intervención realizada respecto a la abstinencia continua a los doce meses.

\section{Discusión}

Los datos finales tras un año de seguimiento indican que no existen diferencias entre las dos intervenciones para conseguir la abstinencia continua al tabaquismo tras un año de seguimiento, y que casi un tercio del total de adolescentes presentan una abstinencia continua, lo que avala la eficacia de la intervención motivacional para conseguir el cese del consumo de tabaco en adolescentes (Heckman et al., 2010; Hettema y Hendricks, 2010; Lai, Cahill, Qin y Tang, 2010). Las intervenciones motivacionales y la incorporación de elementos sensibles al estadio del cambio se postulan como elementos de gran valor en la intervención en un amplio rango de conductas adictivas (Lai et al., 2010; Miller y Rollnick, 1999; Rivera et al., 2008). La inclusión de los motivos para no fumar en estas intervenciones $y$, sobre todo, su disonancia con otras creencias esgrimidas por el adolescente para continuar consumiendo parecen ser un elemento que favorece el cese del consumo de tabaco (Kleinjan et al., 2009). Este tipo de intervención se convierte en una herramienta semiestructurada que favorece la disminución de la ansiedad, así como la percepción del conflicto frente al síndrome de abstinencia, aumenta la motivación positiva y disminuye la incertidumbre como elemento de máximo estrés frente a la decisión de paso al acto (Fuentes, Calatayud, López y Castañeda, 2005).

Además del uso de las disonancias cognitivas para conseguir una abstinencia continua transcurridos doce meses, se presentan algunas técnicas útiles que pretenden facilitar el objetivo del cese del tabaquismo: la inclusión de elementos sensibles al estadio de cambio (Grimshaw y Stanton, 2007; Moreno, 2008), la realización de la consulta en el propio centro escolar (Barrueco et al., 2005; Hodder et al., 2011; Soria-Esojo et al., 2005) y la introducción de elementos motivacionales (Kleinjan et al., 2009; Knight, McGowan,
Dickens y Bundy, 2006; Lai et al., 2010). El uso de recordatorios mediante el correo electrónico o los mensajes de texto al teléfono móvil es otra herramienta terapéutica que ayuda al adolescente en la consecución de una abstinencia continua (Free et al., 2011), siendo internet una de las vías preferidas por los adolescentes para recibir información (ESTUDES, 2010).

Una medida absoluta de cesación es prácticamente imposible porque requeriría un seguimiento de por vida de los sujetos, por lo que al menos el seguimiento debe durar seis meses (Grimshaw y Stanton, 2007; Lai et al., 2010). El presente trabajo informa de los resultados del cese del tabaquismo en puntos de seguimiento múltiples $y$, al igual que en otros estudios, se comprueba cómo el efecto disminuye progresivamente durante este período (Grimshaw y Stanton, 2007; Myers y Brown, 2005) de forma que la reincidencia temprana es un peligro obvio. De los tres controles realizados tras la intervención, la medición del consumo a los doce meses es el mejor indicador ya que evita los sesgos del consumo intermitente típico del tabaquismo adolescente (Burton et al., 2009; Grimshaw y Stanton, 2007; Hughes et al., 2003; Johnson et al., 2009). La respuesta verbal sobre la abstinencia que dan los participantes se ha controlado con la realización de cooximetrías en cada visita de control, con un buen resultado por parte de los no fumadores al obtener en todos los casos un resultado compatible con el cese del consumo. Por tanto, el criterio de respuesta subjetiva tendría una alta fiabilidad per se, siendo útil la determinación del monóxido de carbono como un elemento motivador más que como una forma de comprobar la abstinencia (Barrueco et al., 2005). Aunque algunos autores indican la necesidad de una validación bioquímica de la respuesta subjetiva de los adolescentes sobre el cese o la continuación del consumo, no parece ser una medida muy sensible en los fumadores irregulares (Grimshaw y Stanton, 2007).

La intervención motivacional breve se presenta como la más eficiente para un programa de deshabituación tabáquica en adolescentes, permitiendo al facultativo en una sola sesión tener el mismo éxito con el adolescente fumador que si realiza cuatro sesiones. La falta de diferencias entre las intervenciones motivacionales breve e intensiva podría deberse a existir una menor diferencia entre la eficacia de ambas intervenciones de la estimada para calcular el tamaño de muestra, o bien por ser necesario incrementar el reclutamiento de adolescentes fumadores, como ocurre con la mayoría de estudios realizados sobre esta población (Grimshaw y Stanton, 2007). A diferencia de lo que ocurre en los adultos, quienes racionalizan más el hábito tabáquico con una disminución del número de disonancias y una justificación de manera más consistente de su comportamiento (Kleinjan et al., 2009), los adolescentes presentan un mayor número de disonancias y una menor racionalización de las mismas. Esto puede explicar el alto porcentaje de jóvenes que desean dejar de fumar y la necesidad de emplear menos tiempo en las intervenciones a realizar por los médicos (Pérez-Milena et al., 2006; Pericas et al., 2009). 
Otras intervenciones complejas, como el counselling, la intervención psicosocial, las derivadas de la teoría del aprendizaje y el modelado social o diversas terapias farmacológicas, tampoco aprecian diferencias entre modelos breves 0 intensivos (Barrueco et al., 2007; Grimshaw y Stanton, 2007; NICE, 2008). La intervención intensiva permite disponer de mayor tiempo para revisar y profundizar en las disonancias cognitivas, por lo que puede ser más útil en aquellos adolescentes de mayor edad y que presenten una menor motivación para dejar de fumar (Barrueco et al., 2007; Grimshaw y Stanton, 2007). La reducción progresiva del número de cigarrillos es una técnica que se introduce de forma diferenciadora en esta intervención, pero parece producir tasas de abandono equivalentes al hecho de dejarlo abruptamente sin reducción previa, como ocurre en fumadores adultos (Lindson et al., 2010), por lo que su efecto en la abstinencia en adolescentes fumadores con un patrón de consumo irregular puede ser pobre.

Se ha intentado evitar el sesgo originado en la variabilidad en las intervenciones de los diferentes profesionales mediante una formación previa común realizada por un médico de familia especialista en entrevista clínica y el empleo de un guión común para el desarrollo de cada intervención. Aunque no se han encontrado datos que indiquen heterogeneidad en las intervenciones realizadas por cada médico/a de familia, será preciso valorar en un futuro las influencias aumentando el tamaño de muestra obtenido por algunos facultativos.

La mayoría de estudios sobre el tabaquismo en adolescentes han demostrado la importancia de dejar de fumar antes de los 30 años para evitar la mortalidad relacionada con el tabaco, pero se ha prestado poca atención al desarrollo de intervenciones para dejar de fumar en la población adolescente basadas en la evidencia. Las particularidades diferenciadoras del tabaquismo en la adolescencia, sobre todo su consumo intermitente (ESTUDES, 2010; Pérez-Milena et al., 2007), no debe hacer olvidar el riesgo acompañante y la existencia de dependencia a la nicotina aún en estadios iniciales de consumo (Barrueco et al., 2007; Grimshaw y Stanton, 2007). Es por ello que son necesarias acciones de prevención secundaria (Ramos y Mayayo, 2007) para evitar este problema de salud pública. En España más de la mitad de los adolescentes estaría dispuesto a dejar de fumar (Barrueco et al., 2005; ESTUDES, 2010; Pérez-Milena et al., 2006) lo que supone una magnífica oportunidad para evitar que sean adultos fumadores en un futuro cercano. La integración de intervenciones de deshabituación tabáquica en los programas de prevención del tabaquismo que se realizan en los centros escolares, así como la implicación del profesorado y los padres, posibilitaría la utilización de este instrumento de tratamiento del tabaquismo en jóvenes, más allá del marco limitado de la consulta (Barrueco et al., 2007). Con los datos obtenidos podemos concluir que una intervención motivacional realizada por médicos de familia en el propio instituto puede ser clave para obtener mejores resultados en el cese del tabaquismo en adolescentes y que la intervención breve en la población adolescente sería la más coste-eficaz (Grimshaw y Stanton, 2007; Heckman et al., 2010; Hettema y Hendricks, 2010). Sigue siendo necesario realizar estudios con un mayor tamaño de muestra y un seguimiento a largo plazo para conocer el perfil del adolescente que se beneficiaría de una intervención motivacional intensiva.

\section{Reconocimientos}

A todo el profesorado perteneciente a los Institutos de Educación Secundaria, en especial a los departamentos de Orientación, que con su ayuda han hecho posible las intervenciones en los centros educativos. Al personal de la Fundación de Investigación Biosanitaria Alejandro Otero de Jaén, por su ayuda metodológica. A Juan Manuel López Ángeles, por su ayuda en la redacción en inglés.

El proyecto de investigación ha recibido una subvención para la financiación de la Investigación Biomédica y en Ciencias de la Salud en Andalucía (código PI 0160/2008, Secretaria General de Calidad y Modernización de la Junta de Andalucía, BOJA n 12 de 20 de enero de 2008).

\section{Conflicto de intereses}

Los autores no tienen conflictos de intereses que declarar.

\section{Referencias}

Barrueco, M., Jiménez, C., Palomo, L., Torrecilla, M., Romero, P. y Riesco, J. A. (2005). Veracidad de la respuesta de los fumadores sobre su abstinencia en las consultas de deshabituación tabáquica. Archivos de Bronconeumología, 41, 135-40. doi: 10.1016/S15792129(06)60414-X.

Barrueco, M., Gómez, G., Torrecilla, M., Pérez, A. y Bartolomé, C. (2007). Valor de la intervención breve y los tratamientos farmacológicos para dejar de fumar en adolescentes. Archivos de Bronconeumología, 43, 334-0. doi:10.1016/S15792129(07)60079-2.

Bellón Saameño, J. A., Delgado Sánchez, A., Luna del Castillo, J. D. y Lardelli Claret, P. (1996a). Validez y fiabilidad del cuestionario de función familiar Apgar familiar. Atención Primaria, 18, 289-96.

Bellón Saameño, J. A., Delgado Sánchez, A., Luna del Castillo, J. D. y Lardelli Claret, P. (1996b). Validez y fiabilidad del cuestionario de apoyo social funcional Duke-UNC-11. Atención Primaria, 18, 15363.

Burton, D., Chakravorty, B., Weeks, K., Flay, B. R., Dent, C., Stacy, A., Sussman, S. (2009). Outcome of a tobacco use cessation 
randomized trial with high-school students. Substance Use \& Misuse, 44, 965-80. doi: 10.1080/10826080802490766

Delegación del Gobierno para el Plan Nacional sobre Drogas. (2010) Encuesta Estatal sobre uso de Drogas en Enseñanzas Secundarias (ESTUDES) 2010. Madrid, Ministerio de Sanidad y Política Social, España.

Free, C., Knight, R., Robertson, S., Whittaker, R., Edwards, P., Zhou, W. y Roberts, I. (2011). Smoking cessation support delivered via mobile phone text messaging (txt2stop): a single-blind, randomised trial. Lancet, 378, 49-55. doi: 10.1016/S0140-6736(11)60701-0

Fuentes Pila, J. M., Calatayud, P., López, E. y Castañeda, B. (2005). La entrevista motivacional: llave del proceso de cambio en la dependencia nicotin-tabáquica. Trastornos Adictivos, 7, 153-65.

Grimshaw, G. M. y Stanton, A. (2007). Intervenciones para el abandono del hábito de fumar en personas jóvenes (Revisión Cochrane traducida). En: La Biblioteca Cochrane Plus, Número 4. Oxford: Update Software Ltd. Disponible en: http://www.update-software. com. (Traducida de The Cochrane Library, 2007 Issue 4. Chichester, UK: John Wiley \& Sons, Ltd.).

Heckman, C. J., Egleston, B. L. y Hofmann, M. T. (2010). Efficacy of motivational interviewing for smoking cessation: a systematic review and meta-analysis. Tobacco Control, 19, 410-6. doi:10.1136/tc.2009.033175

Hettema, J. E. y Hendricks, P. S. (2010). Motivational interviewing for smoking cessation: a meta-analytic review. Journal of Consulting and Clinical Psychology, 78, 868-84.

Hodder, R. K., Daly, J., Freund, M., Bowman, J., Hazell, T. y Wiggers, J. (2011). A school-based resilience intervention to decrease tobacco, alcohol and marijuana use in high school students. BMC Public Health, 11, 722. doi:10.1186/1471-2458-11-722.

Hughes, J. R., Keely, J. P., Niaura, R. S., Ossip-Klein, D. J., Richmond, R. L. y Swan, G. E. (2003). Measures of abstinence in clinical trials: issues and recommendations. Nicotine \& Tobacco Research, 5, 13-25. doi:10.1093/ntr/5.1.13

Infante, C. y Rubio-Colavida, J. M. (2004). La prevalencia del consumo de tabaco en España. Adicciones, 16 (Sup/ 2), 59-73.

Johnson, C. C., Myers, L., Webber, L. S., Boris, N. W., He, H. y Brewer, D. (2009). A school-based environmental intervention to reduce smoking among high school students: the Acadiana Coalition of Teens against Tobacco (ACTT). Internal Journal of Environmental Research and Public Health, 6, 1298-316. doi:10.3390/ ijerph6041298.

Kleinjan, M., van den Eijnden, R. J. y Engels, R. C. (2009). Adolescents' rationalizations to continue smoking: the role of disengagement beliefs and nicotine dependence in smoking cessation. Addictive Behaviors, 34, 440-5. doi:10.1016/j.addbeh.2008.12.010.

Knight, K. M., McGowan, L., Dickens, C. y Bundy, C. (2006). A systematic review of motivational interviewing in physical health care settings. British Journal of Health Psychology, 11, 319-32. doi: 10.1348/135910705X52516.
Lai, D. T. C., Cahill, K., Qin, Y. y Tang, J. L. (2010). Intervenciones motivacionales para el abandono del hábito de fumar (Revision Cochrane traducida). En: Biblioteca Cochrane Plus Número 1. Oxford: Update Software Ltd. Disponible en: http://www.updatesoftware.com (Traducida de The Cochrane Library, 2010 Issue 1 Art no. CD006936. Chichester, UK: John Wiley \& Sons, Ltd.).

Lando, H. A., Hipple, B. J., Muramoto, M., Klein, J. D., Prokhorov, A. V., Ossip, D. J. y Winickoff, J. P. (2010). El tabaco es un problema que afecta a los niños en el mundo entero. Boletín de la Organización Mundial de la Salud, 88, 2. URL: http://www.who.int/bulletin/ volumes/88/1/09-069583/es/index.html [17/05/2012].

Lindson, N., Aveyard, P. y Hughes, J. R. (2010). Reducción versus cese abrupto en fumadores que quieren dejar el hábito. (Revisión Cochrane traducida). En: Biblioteca Cochrane Plus Número 3. Oxford: Update Software Ltd. Disponible en: http://www.updatesoftware.com [17/05/2012].

Moreno Mariscal, S. (2008). Guía de práctica clínica de adicción al tabaco. Consejería de Igualdad y Bienestar Social, Junta de Andalucia. URL: http://www.juntadeandalucia. es/igualdadybienestarsocial/opencms/system/bodies/ Drogodependencia/Publicacion/GUIA_CLINICA_TABACO/GUIA_ CLINICA_TABACO.pdf [18/05/2012].

Miller, W. R. y Rollnick, S. (1999). La entrevista motivacional: preparar el cambio de conductas adictivas. Barcelona: Paidós Ibérica. (trabajo original publicado en 1991).

Myers, M. G. y Brown, S. A. (2005). A controlled study of a cigarette smoking cessation intervention for adolescents in substance abuse treatment. Psychology of Addictive Behaviors, 19, 230-3.

National Institute for Health and Clinical Excellence (2008). Brief interventions and referral for smoking cessation in primary care and other settings. URL: http://www.nice.org.uk/nicemedia/pdf/ PH001_smoking_cessation.pdf [18/05/2012].

Pérez-Milena, A., Martínez-Fernández, ML., Pérez-Milena, R., Jiménez-Pulido, I., Leal-Helmling. F. J. y Mesa-Gallardo, I. (2006). Tabaquismo y adolescentes: ¿buen momento para dejar de fumar? Relación con factores sociofamiliares. Atención Primaria, 37, 4526. doi: $10.1157 / 13088886$.

Pérez-Milena, A., Leal, F. J., Jiménez, I., Mesa, M. I., Martínez M. L. y Pérez-Milena R. (2007). Evolución del consumo de sustancias tóxicas entre los adolescentes de una zona urbana (1997-2004). Atención Primaria, 39, 299-304. doi: 10.1157/13106285.

Pérez-Trullén, A., Herrero, I., Clemente, M. L. y Escosa, L. (2004). Abordaje de la prevención y el tratamiento del tabaquismo: ¿a quién, cuándo y cómo realizar la deshabituación tabáquica? Archivos de Bronconeumología, 40(Sup/3), 63-73.

Piñeiro, B., Míguez, M. C. y Becoña, E. (2010). La entrevista motivacional en el tratamiento del tabaquismo: una revisión. Adicciones, 22, 353-64.

Ramos, A. y Mayayo, M. L. (2007). Manual SEPAR de procedimientos: Abordaje del tabaquismo. Barcelona: SEPAR. 
Rivera, S., Villouta, M. F. e llabaca, A. (2008) Entrevista motivacional: ¿cuál es su efectividad en problemas prevalentes de la atención primaria? Atención Primaria, 40, 257-61. doi: 10.1157/13120020.

Sociedad Española de Medicina Familiar y Comunitaria. (2007) El papel de la Atención Primaria ante los problemas de salud relacionados con el consumo de drogas. Barcelona. URL: http://www.pnsd. msc.es/Categoria2/publica/pdf/AtencionPrimariaDrogas.pdf [20/05/2012].

Soria-Esojo, M. C., Velasco-Garrido, J. L., Hidalgo-Sanjuán, M. V., Luiz-Martínez, G., Fernández-Aguirre, C. y Rosales-Jaldo, M. (2005). Intervención sobre tabaquismo en estudiantes de enseñanza secundaria de la provincia de Málaga. Archivos de Bronconeumología, 41, 654-8. doi: 10.1016/S15792129(06)60332-7. 
